\title{
SPE 119104
}

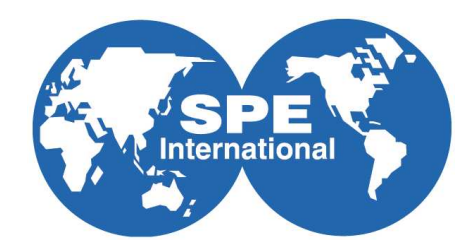

\section{A Multiscale Mixed Finite-Element Method for Vuggy and Naturally-Fractured Reservoirs Astrid Fossum Gulbransen, Vera Louise Hauge, and Knut-Andreas Lie, SINTEF ICT}

Copyright 2009, Society of Petroleum Engineers

This paper was prepared for presentation at the 2009 SPE Reservoir Simulation Symposium held in The Woodlands, Texas, USA, 2-4 February 2009.

This paper was selected for presentation by an SPE program committee following review of information contained in an abstract submitted by the author(s). Contents of the paper have not been reviewed by the Society of Petroleum Engineers and are subject to correction by the author(s). The material does not necessarily reflect any position of the Society of Petroleum Engineers, its officers, or members. Electronic reproduction, distribution, or storage of any part of this paper without the written consent of the Society of Petroleum Engineers is prohibited. Permission to reproduce in print is restricted to an abstract of not more than 300 words; illustrations may not be copied. The abstract must contain conspicuous acknowledgment of SPE copyright.

\begin{abstract}
Vugs, caves, and fractures can significantly alter the effective permeability of carbonate reservoirs and should be accurately accounted for in a geomodel. Accurate modeling of the interaction between free-flow and porous regions is essential for flow simulations and detailed production engineering calculations. However, flow simulation of such reservoirs is very challenging because of the co-existence of porous and free-flow regions on multiple scales that need to be coupled.

Multiscale methods are conceptually well-suited for this type of modeling as they allow varying resolution and provide a systematic procedure for coarsening and refinement. However, to date there are hardly no multiscale methods developed for problems with both free-flow and porous regions. Herein we develop a multiscale mixed finite-element (MsMFE) method for detailed modeling of vuggy and naturally-fractured reservoirs as a first step towards a uniform multiscale, multiphysics framework. The MsMFE method uses a standard Darcy model to approximate pressure and fluxes on a coarse grid, whereas fine-scale effects are captured through basis functions computed numerically by solving local Stokes-Brinkman flow problems on the underlying fine-scale geocellular grid. The Stokes-Brinkman equations give a unified approach to simulating free-flow and porous regions using a single system of equations, avoid explicit interface modeling, and reduce to Darcy or Stokes flow in certain parameter limits.

In the paper, the MsMFE solutions are compared with fine-scale Stokes-Brinkman solutions for test cases including both short- and long-range fractures. The results demonstrate how fine-scale flow in fracture networks can be represented within a coarse-scale Darcy flow model by using multiscale elements computed solving the Stokes-Brinkman equations. The results indicate that the MsMFE method is a promising path toward direct simulation of highly detailed geocellular models of vuggy and naturally-fractured reservoirs.
\end{abstract}

\section{Introduction}

Naturally fractured and carbonate reservoirs are composed of porous material, but will typically also contain relatively large void spaces in the form of fractures, small cavities, and caves, which are called vugs in the geological literature. Flow simulation of such formations is very challenging because of the co-existence of porous and free-flow domains on multiple scales that require coupling (Wu et al. 2006).

The Darcy-Stokes equations have been used to model industrial infiltration processes and coupled surface and subsurface flow, for which the porous and the free-flow domains are well separated. The Darcy-Stokes model consists of Darcy's law combined with mass conservation in the porous subdomain and the Stokes equations in the free-flow subdomain. To close the model, one must specify conditions on the interface between the Darcy and Stokes subdomains. All such conditions require continuity of mass and momentum over the interface, but differ in the way they allow the tangential component to jump across the interface.

In carbonate reservoirs, the porous and free-flow domains are not well separated: vugs and rock matrix are intertwined throughout the reservoir, often on multiple scales. This means that the coupled Darcy-Stokes approach is not feasible for several reasons. First of all, it would require precise information about the location and geometry of the interface between vugs and the porous matrix and experimentally determined values related to the interface conditions. Whereas this information can be obtained for an engineered medium or a small rock sample, is generally not possible to obtain for a sector or a full reservoir model. Second, explicit representation of the medium on a centimeter scale, as required to resolve vugs and fractures, would make the flow problem computationally intractable. Finally, the free-flow domains may contain loose fill-in material or particle suspensions in the fluids filling the void space. 
Hence, when the extent of the hydrocarbon reservoir rock is large, it is not reasonable to apply the Darcy-Stokes equations for the whole domain. Instead, Arbogast and Lehr (2006) predicted, using tools from homogenization theory, that the appropriate macroscopic model would be of Darcy type, with effective macroscale permeability derived through upscaling by solving the Darcy-Stokes equations on the microscale within an upscaling block. To treat the block problems, Arbogast and Brunson (2007) designed a mixed finite-element formulation with a single set of basis functions that apply for the entire Darcy-Stokes system. (See also Karper et al. (2009) for an alternative unified discretization). Arbogast and Gomez (2009) recently extended this approach to three spatial dimensions and developed new and efficient multigrid solvers to solve the resulting highly ill-conditioned saddle-point linear system.

Another upscaling approach was recently presented by Popov et al. (2007, 2009), in which the Stokes-Brinkman equations, rather than the Darcy-Stokes equations, are used on the fine scale to compute upscaled effective permeabilities. The StokesBrinkman equations can be reduced to either the Stokes or the Darcy equations in appropriate parameter limits and give a somewhat coarser model that does not require a precise description of the interface between free-flow and porous domains. This is advantageous for applications to real media for which the location of the vug boundaries is uncertain and for which resolving the Stokes part of the flow is computationally intractable. Moreover, the Stokes-Brinkman model opens up for a seamless transition between Darcy and Stokes, which may be appropriate in damaged zones, etc. Also, from a numerical point-of-view it is attractive to use a single model in which the two domains are represented implicitly through the parameters instead of a two-domain approach involving explicit modeling of the interface between the vuggy and porous domains.

Common for both the upscaling approaches discussed above is that they use a computation on a fine scale to predict effective properties on a coarser scale. Multiscale simulation (Hou and Wu 1997; Arbogast and Bryant 2002; Chen and Hou 2003; Jenny et al. 2003) is an alternative approach that is conceptually well-suited for this type of modeling, as it enables varying resolution and provides a systematic procedure for coarsening and refinement. For Darcy flow, multiscale methods have proved to be more robust than standard upscaling methods (Kippe et al. 2008) and have the advantage that they offer subscale resolution and thus can be used as highly efficient approximate solvers for direct solution of the full fine-scale problem. Multiscale methods have proved capable of handling industry-standard complexity both with respect to grid representation (Aarnes et al. 2008) and flow physics (Lee et al. 2008; Zhou and Tchelepi 2008; Hajibeygi et al. 2008; Krogstad et al. 2009). Natvig et al. (2009) recently demonstrated how multiscale methods can be used to simulate geological models with fracture corridors modeled as volumetric objects with high permeability. To date, however, multiscale methods have not been applied to simulate flow in naturally-fractured and vuggy media using a multiphysics approach with different flow models on the fine and the coarse scale.

This paper presents a multiscale mixed finite-element (MsMFE) method (Chen and Hou 2003; Arbogast and Bryant 2002; Aarnes 2004) for detailed multiphysics simulation of single-phase flow in naturally-fractured and vuggy reservoirs. The MsMFE method uses a standard Darcy model to approximate pressure and velocity on a coarse grid. Fine-scale effects are captured through basis functions that are determined by solving local Stokes-Brinkman flow problems numerically on the underlying finescale geocellular grid. The local flow problems are set up in a way that forces a unit flow across the interface between two coarse blocks, meaning that the corresponding basis functions reduce to the lowest-order Raviart-Thomas (RT0) basis functions for the special case of Darcy flow in a homogeneous medium. In the general case, the basis functions account for local variations of flow velocity caused by subgrid heterogeneities in the porous domains, increased flow velocities resulting from free-flow domains present on the subgrid scale, and geometrical effects in the case of non-square blocks.

The outline of the paper is as follows: We start by introducing the Darcy-Stokes and Stokes-Brinkman models in more detail and discuss how to discretize the latter. We then introduce the MsMFE method and assess its utility through a few illustrative numerical experiments, before we round up the paper with some concluding remarks.

\section{Mathematical Models}

Incompressible flow in a porous rock matrix typically obeys Darcy's law and is described by a first-order elliptic system in which Darcy's law is combined with a mass-conservation equation to relate the pressure $p_{D}$ and the total (interstitial) velocity $\vec{u}_{D}$,

$$
\mu \mathbf{K}^{-1} \vec{u}_{D}+\nabla p_{D}=0, \quad \nabla \cdot \vec{u}_{D}=f
$$

Here, $\mu$ is the fluid viscosity, $\mathbf{K}$ is the permeability of the porous medium, $f$ denotes fluid sources, and we have neglected any body forces. Eq. 1 may alternatively be manipulated to give a second-order elliptic equation. Incompressible flow in open domains, on the other hand, obeys the Stokes equations,

$$
-\mu \nabla \cdot\left(\nabla \vec{u}_{S}+\nabla \vec{u}_{S}^{\top}\right)+\nabla p_{S}=0, \quad \nabla \cdot \vec{u}_{S}=f
$$

The Stokes-Brinkman equations combine Eqs. 1 and 2 into a single equation,

$$
\mu \mathbf{K}^{-1} \vec{u}+\nabla p-\tilde{\mu} \Delta \vec{u}=0, \quad \nabla \cdot \vec{u}=f,
$$

where $p$ is the pressure, $\vec{u}$ is the velocity field, $\mathbf{K}$ is a permeability tensor that is equal to the Darcy permeability in the porous subdomain, $\mu$ is the viscosity of the fluid, and $\tilde{\mu}$ is an effective viscosity. This model gives a unified approach to model flow in both the free-flow and the porous subdomains using a single system of equations. In the free-flow (or fluid) domain, we may 
let $\mathbf{K}$ tend to infinity and set the effective viscosity equal to the fluid viscosity, $\tilde{\mu}=\mu$ to observe that Eq. 3 simplifies to the Stokes equations, Eq. 2. If $\tilde{\mu}$ is set to zero in the porous domains, Eq. 3 simplifies to the coupled Darcy-Stokes equations, which reintroduces the requirement for interface conditions, etc. We therefore set $\tilde{\mu}$ equal to the physical fluid viscosity $\mu$. Still assuming that the body forces are zero, we may rewrite Eq. 3 as

$$
\nabla p=-\mu \mathbf{K}^{-1} \vec{u}+\tilde{\mu} \Delta \vec{u}
$$

A comparison of the magnitude of the two velocity terms on the right-hand side shows that the first term dominates the second by several orders of magnitude for typical reservoirs. In other words, Eq. 4 can be seen as Darcy's equation with a small viscosity perturbation. Other choices of $\tilde{\mu}$ are also possible and may provide more accurate modeling; see Popov et al. (2009) for a more thorough discussion.

In the following we will study Eqs. 1 and 3 as our flow models on the coarse and fine scale, respectively. For both models, we will assume no-flow or pressure boundary conditions and neglect body forces and wells, so that all flows will be driven by boundary conditions only.

\section{The Multiscale Mixed Finite Element Method}

In this section we start by describing the mixed formulation underlying both the MsMFE method and the discretization of the Stokes-Brinkman equations on the fine scale. Then we introduce the MsMFE formulation and describe in some detail the structure of the associated coarse-scale linear systems.

Fine-Scale Discretization and (Hybrid) Linear System. To solve the Stokes-Brinkman equations (Eq. 3) on the fine scale and the Darcy equations (Eq. 1) on the coarse scale (and on the fine scale for comparison), we will use a mixed finite-element formulation. To this end, we start by assuming that the permeability $\mathbf{K}$ is given as a piecewise constant tensor defined over a regular Cartesian grid in $2 \mathrm{D}$; that is, $\mathrm{K}$ is a constant $2 \times 2$ matrix in each cell $E_{k}$. Then, for both equations we seek a pair of functions $(\vec{u}, p)$ from suitable discrete approximation spaces defined over $\Omega=\bigcup_{k=1}^{N} E_{k}$ such that the function pair $(\vec{u}, p)$ satisfies the variational formulation

$$
\begin{aligned}
b(\vec{u}, \vec{v})-c(p, \vec{v}) & =0, \\
c(\pi, \vec{u}) & =(f, \pi)
\end{aligned}
$$

for all test functions $(\vec{v}, \pi)$ from the same discrete approximation spaces. Here, the bilinear forms are defined as (subscript $D$ for Darcy and $S B$ for Stokes-Brinkman),

$$
\begin{gathered}
b_{D}(\vec{u}, \vec{v})=\int_{\Omega} \mu \vec{v} \cdot \mathbf{K}^{-1} \vec{u} d \Omega, \quad b_{S B}(\vec{u}, \vec{v})=\int_{\Omega} \mu \vec{v} \cdot \mathbf{K}^{-1} \vec{u} d \Omega+\int_{\Omega} \tilde{\mu} \nabla \vec{v} \cdot \nabla \vec{u} d \Omega, \\
c(p, \vec{v})=\int_{\Omega} p \nabla \cdot \vec{v} d \Omega, \quad(f, \pi)=\int_{\Omega} f \pi d \Omega .
\end{gathered}
$$

Next, we describe how the bilinear forms can be used to define discrete systems.

For the Darcy problem (Eq. 1) on the fine grid, we use the lowest-order Raviart-Thomas (RT0) elements, for which the pressure is in $\mathbb{Q}_{0}$ (i.e., piecewise constant) and the velocity has one degree-of-freedom associated with the normal component on the interface between each pair of grid cells. (That is, $\nabla \cdot \vec{v}$ is constant in $E_{i} \cup E_{j}$ and $\vec{v} \cdot \vec{n}$ is constant on $\partial E_{i} \cap \partial E_{j}$ and zero on $\left.\partial\left(E_{i} \cup E_{j}\right)\right)$. On the coarse grid, we will use a set of generalized RT0 basis functions that will be defined in the next subsection. In either case, we end up with an indefinite linear system that has both positive and negative eigenvalues and is generally considered hard to solve. Fortunately, a symmetric, positive-definite system can be obtained by applying a technique called hybridization (Brezzi and Fortin 1991), which we for simplicity first will explain for Darcy flow on the fine scale.

The idea behind the hybrid formulation is to first remove the constraint that the normal velocity must be continuous across cell faces, giving a weak formulation that contains jump terms at the cell boundaries. Continuity of the normal component is then reintroduced using Lagrange multipliers, that is, by adding an extra set of equations, in which the pressure $\lambda$ at the cell faces plays the role of the Lagrange multipliers. This procedure does not change $\vec{u}$ or $p$, but enables the recovery of pressure values at element faces, in addition to inducing the desired change in structure of the discrete linear system. The local equations can now be assembled to form a hybrid system of the form

$$
\left[\begin{array}{ccc}
B & C & D \\
C^{\top} & 0 & 0 \\
D^{\top} & 0 & 0
\end{array}\right]\left[\begin{array}{r}
q \\
-p \\
\lambda
\end{array}\right]=\left[\begin{array}{l}
0 \\
f \\
0
\end{array}\right]
$$

Here $\boldsymbol{q}$ is the vector of the outward fluxes ordered cell-wise (with interior faces appearing twice with opposite sign), $\boldsymbol{p}$ the vector of cell pressures, and $\lambda$ the vector of face pressures (without repetitions). The entries in the matrices are

$$
B_{i j}=\int_{\Omega} \mu \vec{v}_{i} \mathbf{K}^{-1} \vec{v}_{j} d \Omega, \quad C_{i j}=\int_{\Omega} \delta_{j} \nabla \cdot \vec{v}_{i} d \Omega, \quad D_{i j}=\int_{\partial \Omega}\left|\vec{v}_{i} \cdot \vec{n}_{j}\right| d s
$$


where $\vec{v}_{i}, \vec{v}_{j}$ are outward pointing velocity basis functions, $\delta_{j}$ is equal one inside cell number $j$ and zero elsewhere, and $n_{j}$ is the normal of cell face $j$. The matrices $\boldsymbol{B}$ and $\boldsymbol{C}$ are block diagonal. The $\boldsymbol{C}$ matrix has a simple geometrical structure, with one column per cell and one row per face; each column corresponds to a unique cell and has a unit value in all rows corresponding to a face in this cell and zeros elsewhere. Similarly, each column of $\boldsymbol{D}$ corresponds to a unique face and has one (for boundary faces) or two (for interior faces) unit entries corresponding to the index/indices of the face in the cell-wise ordering.

For readers more familiar with finite-volume (or finite-difference) methods, we remark that the hybrid mixed finite-element method can be recast as assembling a set of local relations of the form

$$
\boldsymbol{q}_{E}=\boldsymbol{T}_{E}\left(p_{E}-\boldsymbol{\lambda}_{E}\right)
$$

where $\boldsymbol{q}_{E}$ denotes the vector of outward fluxes on the faces of a cell $E, p_{E}$ the pressure at the cell center, $\boldsymbol{\lambda}_{E}$ the pressures at the cell faces, and $\boldsymbol{T}_{E}$ is the so-called transmissibility matrix, which here is a full matrix.

For the Stokes-Brinkman problem (Eq. 3) we use the Taylor-Hood elements $\mathbb{Q}_{2} / \mathbb{Q}_{1}$ (i.e., bilinear elements in pressure space and biquadratic elements in velocity space), which are a standard set of stable elements for the Stokes equations. The degreesof-freedom for the two-dimensional $\mathbb{Q}_{2} / \mathbb{Q}_{1}$ elements are located at the vertices in the grid for pressure and for velocity they are located at the vertices, at the midpoint of each cell face, and at the cell centers. For completeness, we will take a closer look at the corresponding discrete system. Assuming that the permeability tensor $\mathbf{K}$ is diagonal, we split the velocity $\vec{u}$ into its two spatial components $u_{1}$ and $u_{2}$. Letting $\left\{v_{i}\right\}$ and $\left\{\pi_{i}\right\}$ be the scalar Taylor-Hood basis functions, we can set $u_{k}=\sum_{\Omega_{i}} u_{i k} v_{i}$ and $p=\sum_{\Omega_{i}} p_{i} \pi_{i}$. The mixed system can then be assembled in the form

$$
\left[\begin{array}{ccc}
\boldsymbol{B}_{1} & \mathbf{0} & \boldsymbol{C}_{1} \\
\mathbf{0} & \boldsymbol{B}_{2} & \boldsymbol{C}_{2} \\
\boldsymbol{C}_{1}^{\top} & \boldsymbol{C}_{2}^{\top} & \mathbf{0}
\end{array}\right]\left[\begin{array}{c}
\boldsymbol{u}_{1} \\
\boldsymbol{u}_{2} \\
-\boldsymbol{p}
\end{array}\right]=\left[\begin{array}{l}
\mathbf{0} \\
\mathbf{0} \\
\boldsymbol{f}
\end{array}\right]
$$

where $\boldsymbol{u}_{1}$ and $\boldsymbol{u}_{2}$ are vectors of the two velocity components $u_{i 1}$ and $u_{i 2}$, respectively, and $\boldsymbol{p}$ is the vector of pressure values $p_{i}$. Once again assuming no-flow boundary conditions, the entries in the matrices are

$$
B_{i j, k}=\int_{\Omega} \mu v_{i} K_{c, k k}^{-1} v_{j} d \Omega+\int_{\Omega} \tilde{\mu}\left(\frac{\partial v_{i}}{\partial x_{1}} \frac{\partial v_{j}}{\partial x_{1}}+\frac{\partial v_{i}}{\partial x_{2}} \frac{\partial v_{j}}{\partial x_{2}}\right) d \Omega, \quad C_{i j, k}=\int_{\Omega} \frac{\partial v_{i}}{\partial x_{k}} \pi_{j} d \Omega,
$$

where $k=1,2$ denotes the spatial dimension and $c$ denotes the respective cell.

The MsMFE Formulation. To define the MsMFE discretization, we start by defining the coarse grid. Each block in this grid is defined as a connected collection of cells from the fine grid; that is, block number $i$ is given as $\Omega_{i}=\bigcup_{k=1}^{N_{i}} E_{k}$ for some constant $N_{i}$. In the simplest case, the coarse grid is formed as a uniform partition of the fine Cartesian grid so that each block $\Omega_{i}$ becomes rectangular. Over this coarse grid, we define a discrete approximation space that generalizes RT0: the pressure is approximated by a constant in each block, and for the velocity we use a set of basis functions that are computed numerically and contain subcell resolution. Each basis function represents a unit flow over the interface between two coarse blocks, and hence there is one basis function associated with each pair of blocks in the coarse grid (Aarnes et al. 2006). Let $\Omega_{i j}$ be a neighborhood containing two neighboring blocks $\Omega_{i}$ and $\Omega_{j}$. The basis function associated with the interface $\partial \Omega_{i} \cap \partial \Omega_{j}$ is constructed by solving the following flow problem over $\Omega_{i j}$

$$
\mu \mathbf{K}^{-1} \vec{\psi}_{i j}+\nabla \varphi_{i j}-\tilde{\mu} \Delta \vec{\psi}_{i j}=0, \quad \nabla \cdot \vec{\psi}_{i j}=\left\{\begin{array}{cl}
w_{i}(\vec{x}), & \text { if } \vec{x} \in \Omega_{i}, \\
-w_{j}(\vec{x}), & \text { if } \vec{x} \in \Omega_{j}, \\
0, & \text { otherwise. }
\end{array} \quad \vec{\psi}_{i j} \cdot \vec{n}=0 \text { on } \partial \Omega_{i j}\right.
$$

Here $w_{i}(\vec{x})$ is a weight function that is normalized over $\Omega_{i}$ and whose purpose is to produce a flow with unit average velocity over the interface $\partial \Omega_{i} \cap \partial \Omega_{j}$. To get a conservative method, we must choose $w_{i}$ proportional to $q$ in blocks containing nonzero source terms. In all other blocks, $w_{i}$ may be chosen more freely; the simplest choice is $w_{i}(\vec{x})=1 /\left|\Omega_{i}\right|$. (We will return to $w_{i}$ below.) The no-flow boundary condition is imposed to localize the basis functions.

To motivate this construction of basis functions, let us first consider the case of pure Darcy flow in a 1-D homogeneous domain $(\tilde{\mu}=0$ and $\mathbf{K} \equiv \mathbf{I})$. We define $w_{i} \equiv 1$, and set $\Omega_{i}=[-1,0]$ and $\Omega_{j}=[0,1]$. Then by solving Eq. 8, we obtain $\psi_{i j}=1-|x|$, which coincides with the RT0 velocity basis. When K has subscale variation, solving Eq. 8 gives generalized RT0 basis functions that account for the subscale variations in velocity, given a unit velocity over the corresponding block interface. The construction defined in Eq. 8 is general and can be performed for arbitrary connected collections of cells from the fine grid. If $\Omega_{i}$ and $\Omega_{j}$ are allowed to be polyhedral rather than rectangular, we compute the generalization of the RT0 basis functions to polyhedral blocks (Aarnes et al. 2006). The underlying fine grid can also be fully unstructured as discussed in more detail by Aarnes et al. (2008), who also suggest a set of simple guidelines for how to automatically define good coarse grids.

When the coarse blocks contain free-flow regions, the only change is that we use Stokes-Brinkman equations rather than the Darcy equations to compute the basis functions. Using normalized weight functions that scale with trace $(\mathbf{K})$, we produce basis 

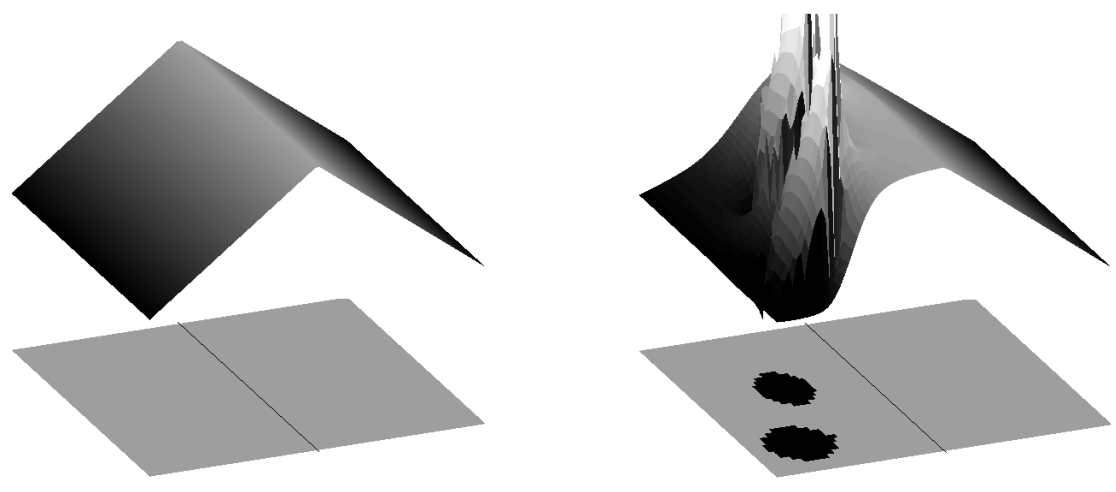

Fig. 1- The x-component of two basis functions for an interface between two rectangular grid blocks for a homogeneous domain with no sources or or sinks (left) and with two high-permeable vugs (right).

functions that correspond to a unit flow over the interface $\partial \Omega_{i} \cap \partial \Omega_{j}$ and account for the increased flow induced by the presence of free-flow regions within the coarse blocks. In the porous subdomains, this choice of $w_{i}$ also means that we avoid unnaturally large velocities in low-permeable parts of the coarse blocks; see Aarnes et al. (2006) for more details. Notice, however, that setting $\tilde{\mu}=\mu$ in Eq. 8 and using Taylor-Hood elements to solve the local flow problem means that we introduce errors in the basis functions for the case of pure Darcy flow. In particular, for homogeneous permeability and rectangular support, we are no longer able to reproduce the RT0 basis functions exactly. Fig. 1 displays basis functions for both a homogeneous and a vugular domain at an interface away from sources/sinks. The left figure shows that the basis function is almost identical to the corresponding RT0 velocity basis, also when solving Stokes-Brinkman equations using $\mathbb{Q}_{2} / \mathbb{Q}_{1}$ elements.

Finally, we mention a few improvements that can be used to increase the accuracy of the multiscale approximation. If $\Omega_{i j}$ is chosen to be larger than $\Omega_{i} \cup \Omega_{j}$, we say that the basis function is computed using overlap or oversampling, which is introduced to lessen the impact of the no-flow boundary condition that localizes the basis function. Similarly, it has been shown that it is advantageous to let the coarse blocks adapt to low-permeable objects like shales (Aarnes et al. 2006) or to long-range high-flow regions like fracture corridors (Natvig et al. 2009). Finally, if a better approximation is required for the pressure, one may also utilize the pressures $\varphi_{i j}$ from the local flow problems, Eq. 8, as additional basis functions; see Krogstad et al. (2009) for more details. Herein, however, our focus is on extending the MsMFE method to a multiphysics setting and we will therefore not use any of these more advanced constructions.

Coarse-Scale Hybrid System. Having constructed the multiscale basis functions, we are now in a position to assemble the global coarse-scale hybrid system. How to do this is thoroughly described by Krogstad et al. (2009) and Skaflestad and Krogstad (2008) for Darcy flow. Here, we will go briefly through the structure of the hybrid system and comment on a few issues that arise because of the fine-scale Stokes-Brinkman model and the use of Taylor-Hood elements.

First, in previous formulations of the MsMFE method, the basis functions have been represented as a vector of fluxes defined on the set of cell-wise ordered faces in the fine grid representing $\Omega_{i j}$ (Krogstad et al. 2009). Using Taylor-Hood to compute basis functions, however, means that the basis functions are given as velocities in nodal points and must therefore be represented as a vector of cell-wise ordered nodal points in the fine grid. A nodal point will typically belong to more than one coarse block and will introduce one new entry for each block it belongs to. In other words, basis functions are represented usinglocal, and not global, nodes, which means that a given nodal point may appear multiple times in the vector of basis functions, each time with a value defined by a unique basis function.

Second, to be able to introduce coarse-grid Lagrange multipliers (interface pressures in the coarse-grid), we need to split the basis functions resulting from solving Eq. 8 in two parts,

$$
\boldsymbol{\psi}_{i j}=\boldsymbol{\psi}_{i j}^{H}-\boldsymbol{\psi}_{j i}^{H}, \quad \boldsymbol{\psi}_{i j}^{H}(E)=\left\{\begin{array}{ll}
\boldsymbol{\psi}_{i j}(E), & \text { if } E \in \Omega_{i j} \backslash \Omega_{j} \\
0, & \text { otherwise }
\end{array} \boldsymbol{\psi}_{j i}^{H}(E)= \begin{cases}-\boldsymbol{\psi}_{i j}(E), & \text { if } E \in \Omega_{j} \\
0, & \text { otherwise }\end{cases}\right.
$$

Next, we arrange all the hybrid basis functions $\boldsymbol{\psi}_{i j}^{H}$ as columns in a matrix $\boldsymbol{\Psi}$. To account for the fact that the basis functions are velocities and not fluxes, we must multiply $\boldsymbol{\Psi}$ with the inverse of the area matrix $\boldsymbol{A}^{-1}$. Then the hybrid multiscale system reads,

$$
\left[\begin{array}{ccc}
\hat{\boldsymbol{\Psi}}^{\top} \boldsymbol{B}^{T H} \hat{\boldsymbol{\Psi}} & \hat{\boldsymbol{\Psi}}^{\top} \boldsymbol{C} \mathcal{I} & \hat{\boldsymbol{\Psi}}^{\top} \boldsymbol{D} \mathcal{J} \\
\mathcal{I}^{\top} \boldsymbol{C}^{\top} \hat{\boldsymbol{\Psi}} & \mathbf{0} & \mathbf{0} \\
\mathcal{J}^{\top} \boldsymbol{D}^{\top} \hat{\boldsymbol{\Psi}} & \mathbf{0} & \mathbf{0}
\end{array}\right]\left[\begin{array}{r}
\boldsymbol{q}^{c} \\
-\boldsymbol{p}^{c} \\
\boldsymbol{\lambda}^{c}
\end{array}\right]=\left[\begin{array}{c}
\mathbf{0} \\
\boldsymbol{f}^{c} \\
\mathbf{0}
\end{array}\right] .
$$



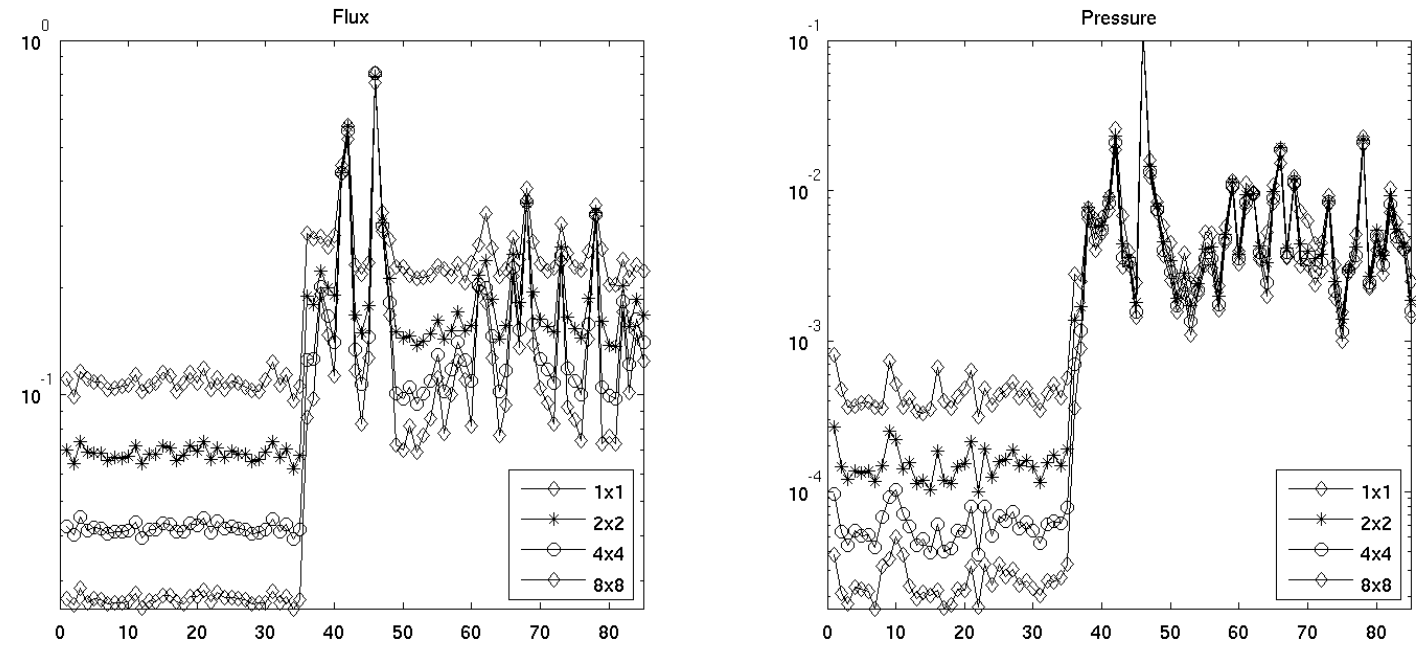

Fig. 2- Discrepancy between the RT0 and $\mathbb{Q}_{2} / \mathbb{Q}_{1}$ solutions measured in a relative $L^{2}$ norm for the 85 layers of the SPE 10 model. For each layer, the discrepancy is measured for the original $60 \times 220$ grid, as well as for $2 \times 2,4 \times 4$, and $8 \times 8$ refinements thereof.

Here, $\hat{\Psi}=\boldsymbol{\Psi} \boldsymbol{A}^{-1}$, the vectors $\boldsymbol{q}^{c}, \boldsymbol{p}^{c}$, and $\boldsymbol{\lambda}^{c}$ contain the coarse-scale degrees-of-freedom, $\mathcal{I}$ is the prolongation from blocks to cells, and $\mathcal{J}$ the prolongation from block faces to cell faces.

The " $C$ sub-matrix" of the coarse system (see Eq. 6) has one column per coarse grid block and one row per coarse face. Each column is associated with a unique coarse block $\Omega_{i j}$ and has a unit entry in all rows corresponding to a coarse face in $\Omega_{i j}$ and zeros elsewhere. Similarly, each column of the " $D$ sub-matrix" is associated with a unique face and has a unit entry in the rows corresponding to the indices of the face in the block-wise ordering. In practice, it is more efficient to construct the $C$ and $D$ sub-matrices geometrically than using the algebraical representation in Eq. 9.

The matrix $\boldsymbol{B}^{T H}$ is the fine-scale analogue of Eq. 6 defined with Taylor-Hood elements rather than RT0, and is a block diagonal matrix of $9 \times 9$ blocks for 2D Cartesian grids. If we use no overlap, the $B$-part of Eq. 9 is block diagonal with respect to the coarse blocks and can be reduced to a symmetric positive-definite system for $\boldsymbol{\lambda}^{c}$. When overlap is used, one is in general better off using a mixed formulation of the system.

Once the solution of Eq. 9 is computed, the fine-scale velocities can be computed as $\boldsymbol{u}=\hat{\boldsymbol{\Psi}} \boldsymbol{q}^{c}$, where one must remember that each nodal point may have contributions from more than one basis function on the coarse grid. To account for this, we average the velocities from all local nodes that coincide with one global node.

\section{Numerical Experiments}

Darcy Flow. Our first test of the multiscale Darcy/Stokes-Brinkman method is on a sandstone reservoir for which the Darcy flow model is applicable. To this end, we will use Model 2 from the 10th SPE Comparative Solution Project (Christie and Blunt 2001). The purpose of this test is threefold:

- calculate the influence of the Stokes part of the Stokes-Brinkman equation for pure Darcy flow;

- compare the Taylor-Hood and Raviart-Thomas elements for pure Darcy flow;

- asses the accuracy of Darcy/Stokes-Brinkman multiscale simulations versus Stokes-Brinkman fine-scale simulations in the porous (matrix) part of a vugular medium.

We start off by determining the influence of the Stokes term in the Stokes-Brinkman equation for a typical Darcy flow. To this end, prescribe a pressure drop of 1 bar in the $y$-direction and no-flow conditions at the left and right boundaries, and compare simulations with $\tilde{\mu}=0$ and $\tilde{\mu}=\mu$ for all 85 layers. The results show that the relative discrepancies in velocity are of the order $10^{-12}$ in all nodal points. The Stokes term can therefore safely be neglected for the SPE 10 model.

In the second test, we compare the discrepancy in face fluxes computed with RT0 and $\mathbb{Q}_{2} / \mathbb{Q}_{1}$ elements. For the RaviartThomas discretization, the fluxes correspond directly to the degrees-of-freedom and will be denoted by $\boldsymbol{q}_{k}^{R T}$ for cell $k$. For the Taylor-Hood discretization, we have to integrate the velocity approximation over the cell faces to derive fluxes $\boldsymbol{q}_{k}^{T H}$. The discrepancies in flux $\left(e_{q}\right)$ and pressure $\left(e_{p}\right)$ are measured using a relative $\mathrm{L}^{2}$ norm:

$$
\left(e_{q}\right)^{2}=\frac{\sum_{k=1}^{N}\left\|\boldsymbol{q}_{k}^{R T}-\boldsymbol{q}_{k}^{T H}\right\|_{2}^{2}}{\sum_{k=1}^{N}\left\|\boldsymbol{q}_{k}^{R T}\right\|_{2}^{2}}, \quad\left(e_{p}\right)^{2}=\frac{\sum_{k=1}^{N}\left\|p_{k}^{R T}-p_{k}^{T H}\right\|_{2}^{2}}{\sum_{k=1}^{N}\left\|p_{k}^{R T}\right\|_{2}^{2}} .
$$




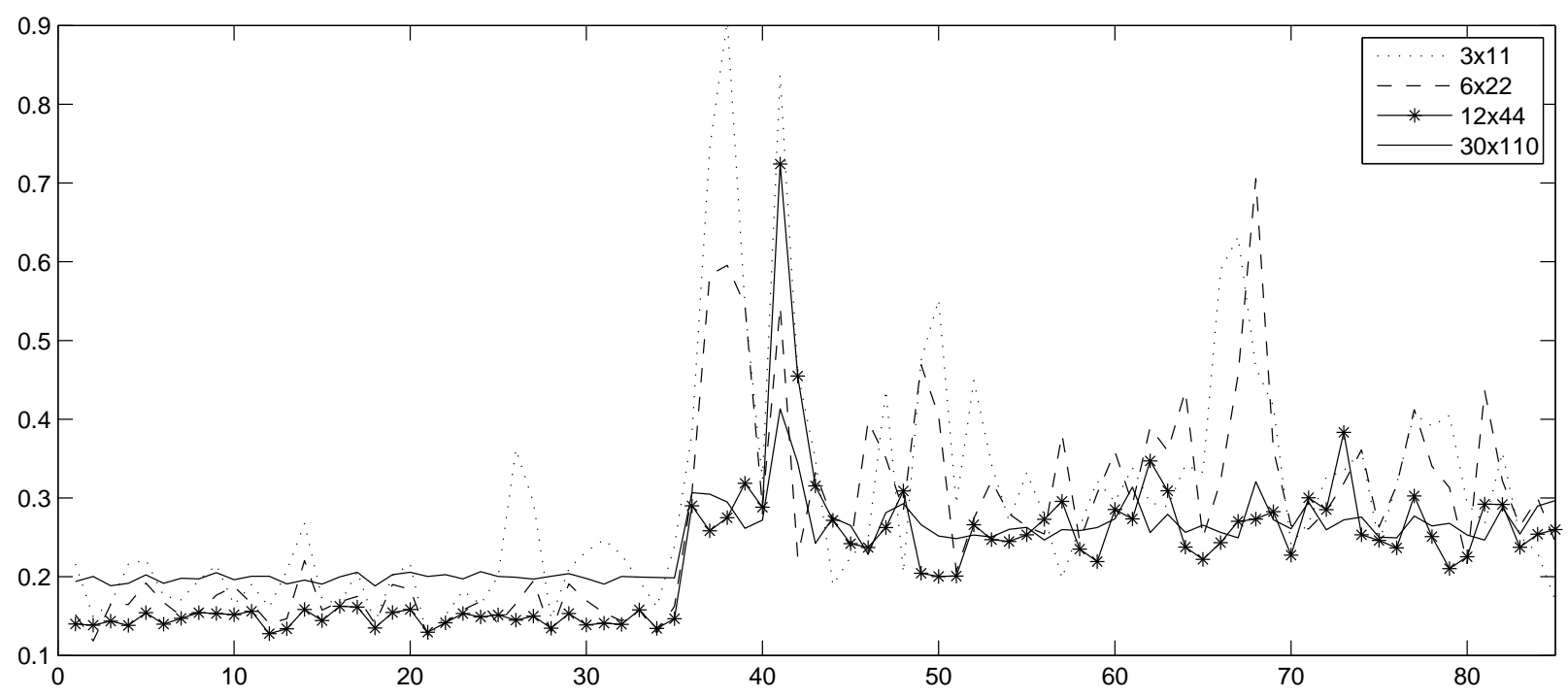

Fig. 3- Discrepancy in velocities for our multiscale solver on four coarse grids relative to the Stokes-Brinkman fine-scale solution for all 85 layers of the SPE 10 model.

Fig. 2 shows these discrepancies for all 85 layers of the SPE 10 model, computed for the original $60 \times 220$ grid, as well as for a $2 \times 2,4 \times 4$, and a $8 \times 8$ refinement of the grid. For the smooth layers of the Tarbert formation, $e_{q}$ oscillates around 0.11 for the original grid and $0.07,0.04$, and 0.03 for the refinements, while for the fluvial bottom layers the discrepancy increases to a mean of $0.27,0.21,0.17$, and 0.16 , respectively. The discrepancy in pressure is significantly lower, with mean values of $4.5 \cdot 10^{-4}, 1.6 \cdot 10^{-4}, 6.0 \cdot 10^{-5}$, and $2.6 \cdot 10^{-5}$ for the top 35 layers, and $8.9 \cdot 10^{-3}, 8.3 \cdot 10^{-3}, 7.7 \cdot 10^{-3}$, and $7.3 \cdot 10^{-3}$ for the lower 50 layers. The results indicate the size of the error associated with the fine-scale solution, which often mistakenly is considered as the correct solution. As expected, the discrepancy between the two schemes decreases with increasing refinement of the grid. However, it is interesting to notice that the pressure does not converge in the same order for the lower fluvial layers as for the smooth top layers of the Tarbert formation.

In the third test, we assess the accuracy of our multiscale Darcy/Stokes-Brinkman method on four different coarse grids. Fig. 3 reports the relative discrepancies in fine-scale velocity obtained from the multiscale method relative to the fine-scale StokesBrinkman solutions for all 85 layers of the model. The discrepancies in velocity are slightly higher than the flux discrepancies in Fig. 2 for the top 35 layers, and approximately at the same level for the 50 layers of the less smooth Tarbert formation. We now select a single layer (Layer 21) from the SPE10 model, and study how the multiscale method performs for different coarse grids. This time we have refined the reference grid by $2 \times 2$. Fig. 4 shows bar plots of the velocity discrepancies for coarse-grid resolutions between $2 \times 2$ coarse blocks (each block consisting of $60 \times 220$ cells) and $120 \times 440$ coarse blocks (each block consisting of 1 cell). It seems that our multiscale methods performs best if the coarse grid blocks have approximately the same number of cells in each direction. Both Fig. 3 and Fig. 4 show that the medium coarse grids generally give better results than the much finer or coarser grids. For this particular problem, the flow patterns are highly influenced by long-range correlations. For coarse grids with few, but large, blocks, the long-range correlations are resolved in the basis functions. For coarse grids with many small blocks, the long-range correlations must be resolved by the global system.

Vuggy and Fractured Reservoirs. The purpose of introducing the Stokes-Brinkman equation in the multiscale method is to capture the influence of free flow in vugs and fractures. To assess how well the fine-scale Stokes-Brinkman flow in vugs and fracture networks is represented within a coarse-scale Darcy flow model using multiscale elements, we consider three simple models. All models have $200 \times 200$ grid cells with each grid cell modeling $10 \times 10 \mathrm{~cm}$. In the multiscale discretization, each domain is divided into $5 \times 5$ coarse grid blocks. The fluid under consideration is water, with $\mu=1 c P$. The flow is driven by a pressure drop of $1 \mathrm{bar}$ in the $x$-direction, and on the lower and upper boundaries we have imposed no-flow conditions.

The first model has 26 randomly distributed vugs with sizes of $1.8-10.4 \mathrm{~m}^{2}$ (diameters of $70-220 \mathrm{~cm}$ ), see Fig. 5. The homogeneous matrix permeability equals 1 Darcy, while the permeability in the vugs is set to be $10^{7}$ times higher. The second model contains fourteen long-range fractures that are straight lines in the vertical and horizontal directions, with apertures of 10 $\mathrm{cm}$ and lengths of 300-900 $\mathrm{cm}$ (see Fig. 6). The third model combines the first two models, getting a vugular medium in which some of the vugs are connected by long-range fractures (see Fig. 7).

Fig. 5 shows the velocities obtained by solving the Stokes-Brinkman directly on the fine-scale model compared with the fine-scale velocities computed by our Darcy/Stokes-Brinkman multiscale method. Streamlines are drawn on top of the velocity plots to better visualize the flow. The model can be characterized as having short correlation lengths in the sense that the each 


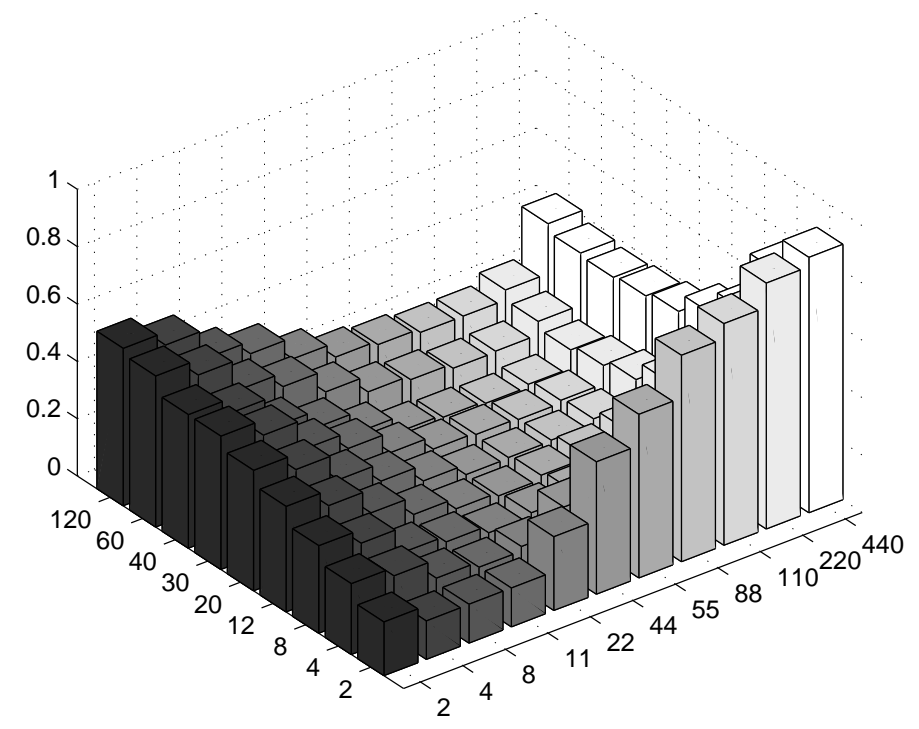

Fig. 4- Multiscale simulations of Layer 21 of the SPE 10 model. The figure compares fine-scale velocities computed directly on a $2 \times 2$ refinement of the original $60 \times 220$ grid with multiscale simulations for various different coarse grids. The numbers on the $x$ - and $y$-axes correspond to the numbers of coarse blocks in the respective directions.

free-flow vug is confined to a single coarse block. Numerous simulations of similar type have shown that the multiscale method is able to reproduce flow of this type with good accuracy. For the model in Fig. 5, the velocity discrepancy is only 0.07 in the relative $\mathrm{L}^{2}$ norm.

The second model has somewhat longer correlation lengths in the form of vertical and horizontal fractures that typically penetrate a few consecutive blocks. (The longest fracture penetrates blocks $(1,2)$ to $(4,2)$, where block $(1,1)$ is in the lower left corner.) The multiscale method is still able to deliver reasonable accuracy, with velocity discrepancies 0.07 in the relative $\mathrm{L}^{2}$ norm, although the streamline plots reveal some qualitative differences. In the third model, some of the vugs are connected by fractures and this gives longer correlations in the form of free-flow regions that extend in both coordinate directions; an example is the continuous free-flow region that extends from block $(3,3)$ to block $(5,1)$. Although the velocity discrepancy only has increased to 0.09 for this model, the qualitative differences are now much easier to spot.

In an effort to explain the larger qualitative discrepancies observed when free-flow regions extend beyond a single block, we focus on coarse block number $(5,3)$, which is the rightmost block in the middle row of Fig. 7 and has the largest discrepancy between the fine-scale and the multiscale solutions. The upper part of Fig. 8 shows the $x$ - and $y$-components of the basis function defined by blocks $(4,3)$ and $(5,3)$ and the lower part of Fig. 8 shows similar plots for the basis function defined by block $(5,3)$ only (with pressure boundary conditions on the right boundary). Since the weight function $w_{i}$ scales with trace( $\left.\mathbf{K}\right)$, the cells in the fracture and the vug will be assigned a weight that is $10^{7}$ time higher than in the surrounding cells, meaning that the sources and sinks in the non-fractured cells in the two-block system are effectively set to zero. In the one-block system, the vug and fracture will also be assigned a weight that is $10^{7}$ time higher than in the surrounding cells, but in addition we have an open boundary at the right hand side. As a result, the flow in block $(4,3)$ is driven through the fracture, and when it enters block $(5,3)$, it continues into the vug and then spreads out towards the open boundary at the right edge. Some of the flow continues down the vertical fracture and is driven towards the right edge from there. In effect, this means that the background flow in the matrix is not represented by this basis function in large parts of the local domain, which leads to the white no-flow region seen in block $(5,3)$ of the multiscale solution in Fig. 7. A similar behavior has been seen in a wide variety of models run by the authors.

For fractures extending beyond a single block in the diagonal direction, numerous experiments demonstrate the opposite effect, namely that the multiscale method introduces large flow in the porous region surrounding the fracture. This can be explained as follows: suppose a fracture connects blocks $(i, j)$ and $(i+1, j+1)$. Since the MsMFE method only represents coarse-scale flow between coarse blocks sharing a common face, the flow from block $(i, j)$ to block $(i+1, j+1)$ must make a detour in blocks $(i+1, j)$ and/or $(i, j+1)$. The same effect has been observed previously for pure Darcy flow and is discussed in more detail by Kippe et al. (2008).

To overcome the problem of fractures (and other high-flow regions) extending beyond a single block, one can introduce extra coarse blocks that represent the long-range fractures. In Fig. 8 this amounts to splitting block $(4,3)$ into three blocks that all are coupled with block $(5,3)$ and together take care of the flow in the fracture and in the porous region above and below the horizontal fracture. The efficiency of this approach has been demonstrated by Natvig et al. (2009), but has so far not been implemented in our simple Darcy/Stokes-Brinkman multiscale solver. 
FS

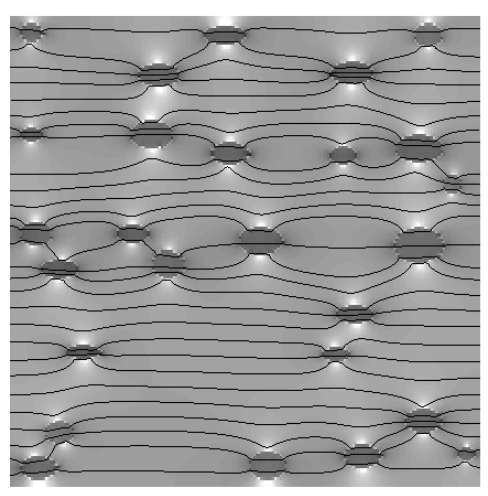

MS

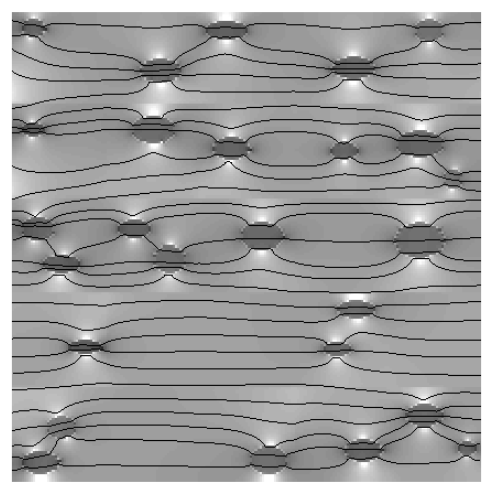

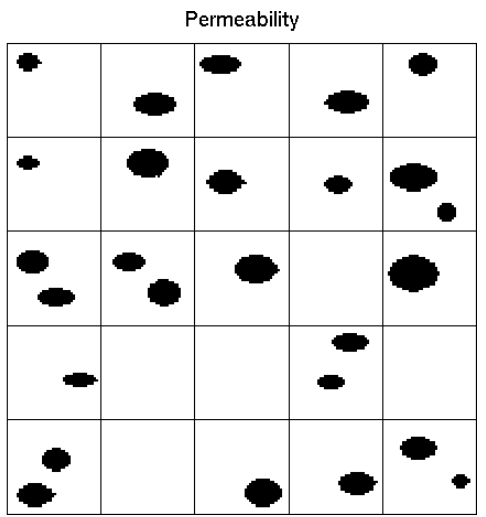

Fig. 5- Simulations of a vuggy reservoir with a homogeneous matrix on a $200 \times 200$ grid. The left and middle plots show the logarithm of the velocity for the fine-scale (FS) and the multiscale (MS) solutions, respectively, overlaid by streamlines. The right plot shows the permeability with high-permeable vugs in black, overlaid by the coarse-grid partitioning.
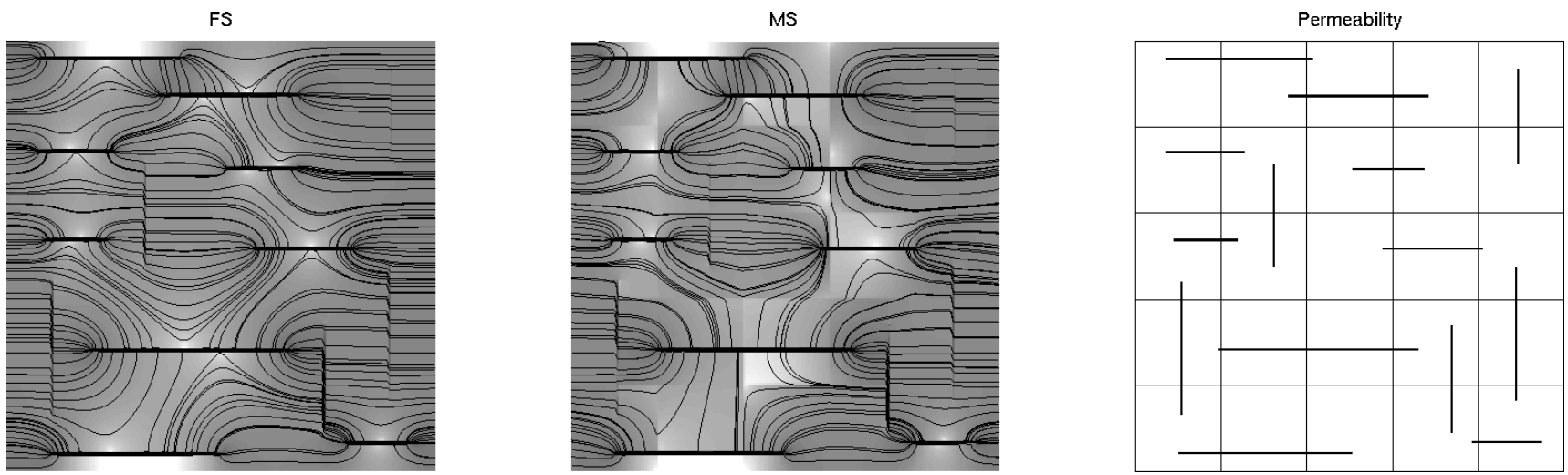

Fig. 6- Simulations of a reservoir with a homogeneous matrix and high permeable fractures on a $200 \times 200$ grid. The left and middle plots show the logarithm of the velocity for the fine-scale (FS) and the multiscale (MS) solutions, respectively, overlaid by streamlines. The right plot shows the permeability with high-permeable fractures in black, overlaid by the coarse-grid partitioning.

FS

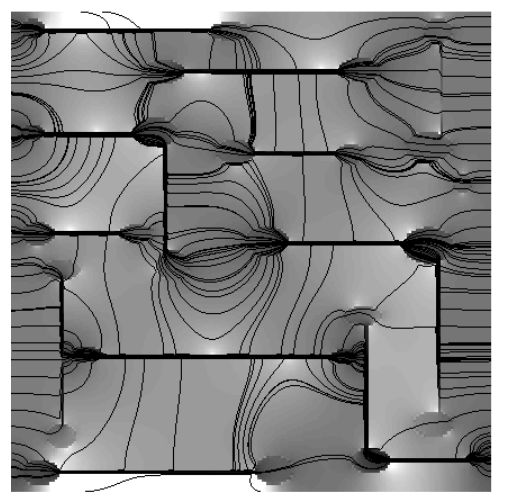

MS

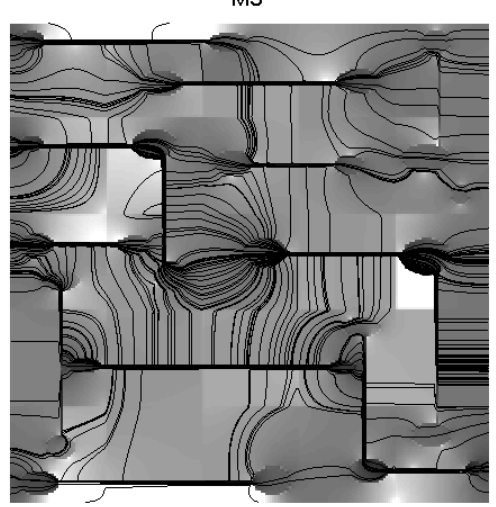

Permeability

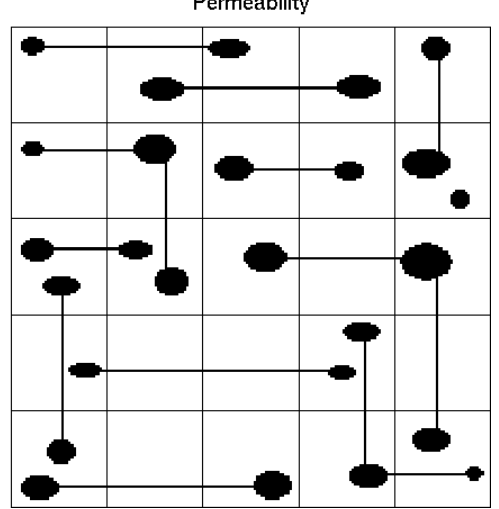

Fig. 7- Simulations of a vuggy reservoir with a homogeneous matrix and high-permeable fractures on a $200 \times 200$ grid. The left and middle plots show the logarithm of the velocity for the fine-scale (FS) and the multiscale (MS) solutions, respectively, overlaid by streamlines. The right plot shows the permeability with high-permeable vugs and fractures in black, overlaid by the coarse-grid partitioning. 

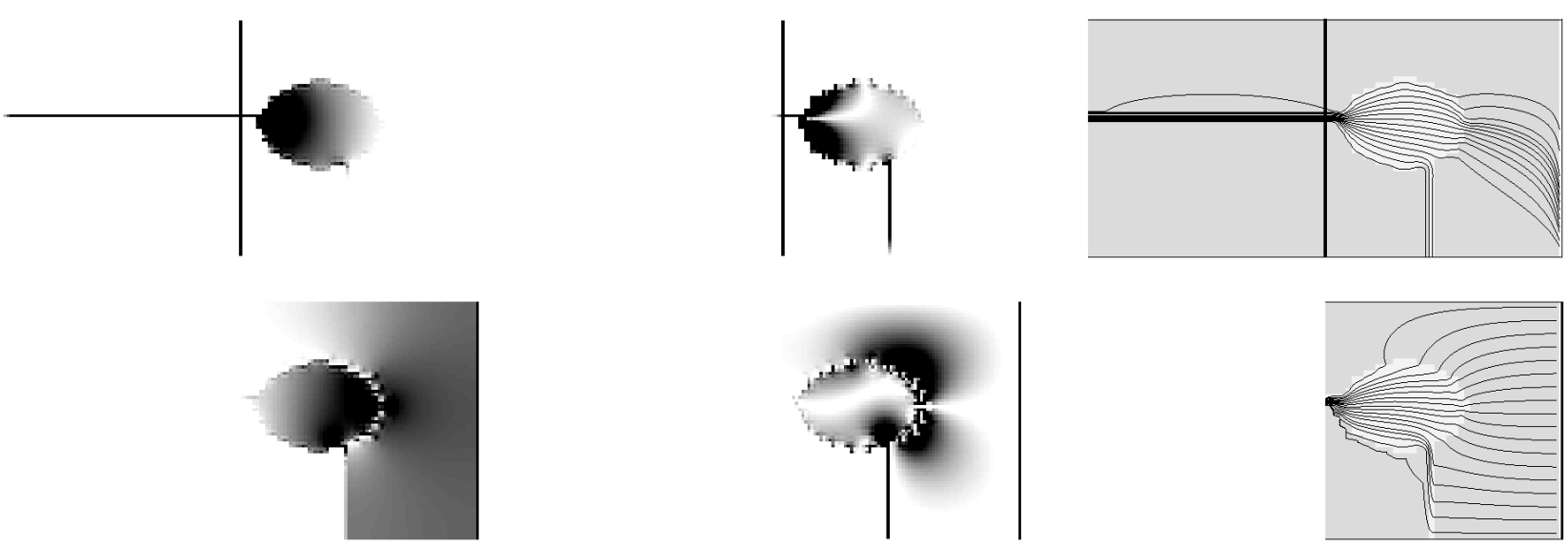

Fig. 8- Basis functions associated with the interface between coarse blocks $(4,3)$ and $(5,3)$ at the top, and basis functions associated with the right boundary face of $(5,3)$ at the bottom. The figures to the left show the basis functions in the $x-$ direction (white is flow in the positive direction, black is flow in the negative direction), the figures in the middle show the basis functions in the y-direction, and to the right we see the permeability overlaid by streamlines.

\section{Concluding Remarks}

In this paper we have developed a multiphysics version of the mixed finite-element method in which the Stokes-Brinkman equations are used to compute basis functions to be used in a Darcy flow model on a coarse scale. The new methodology was applied to two examples. First, we considered a strongly heterogeneous sandstone reservoir (Model 2 from the SPE 10 benchmark), for which we demonstrated that the effect of the second-order term in the Stokes-Brinkman models is negligible and that the discrepancies introduced when using the multiscale method are of the same order-of-magnitude as the discrepancy observed between the Raviart-Thomas and Taylor-Hood discretizations of the fine-scale Darcy equations. In the second example, we considered three simplified 2-D models of fractured and vugular media that are typical for a large number of experiments run by the authors.

In both examples, we observed that the multiscale method delivers qualitatively correct solutions with good accuracy when correlation lengths are small or approximately the size of the coarse blocks (e.g., when free-flow regions are confined to a single coarse block). For cases with long-range correlations (e.g., in the form of free-flow regions that extend beyond a single coarse block), the multiscale method is able to reproduce major parts of the flow patterns, but also introduces large local errors. This is particularly evident for some of the fluvial layers from the SPE 10 benchmark, for which the intertwined high-permeable channels provide a complex combination of short- and long-range correlations that are hard to capture with high pointwise accuracy. Hence, we observe higher $\mathrm{L}^{2}$ errors than for the test cases with vugs and fractures. On the other hand, these (local) errors are not necessarily important when measuring integrated quantities like production curves from wells, etc.

To also get correct small-scale details for cases with complex combinations of short- and long-range correlations, one may either use global boundary conditions for the local flow problems; use oversampling when constructing the basis functions; introduce an adaptive coarsening in which free-flow or high-permeable regions are represented as extra coarse blocks as discussed by Aarnes et al. (2006) for long-correlation shale objects and by Natvig et al. (2009) for fracture corridors; or use an adaptive multiscale method (Nordbotten 2009). This is a topic of ongoing research.

Although the Stokes-Brinkman model is a promising approach for simulating vugular and naturally-fractured reservoirs, its discretization using the Taylor-Hood elements is relatively costly because of the high number of degrees of freedom. Hence, flow problems may fast become computationally intractable with increasing model sizes, in particular in 3-D. The multiscale method developed herein has a natural parallelism in the computation of basis function and also has a potential for reduced memory requirements, and may therefore be an efficient approach for attacking high-resolution 3-D models.

\section{Acknowledgments}

The authors gratefully acknowledge financial support from Shell Norge AS and the Research Council of Norway under grant number 175962/S30. We also thank Peter Popov and Dr. Yalchin Efendiev for helpful discussions. 


\section{Nomenclature}

Physical quantities:

$\mathrm{K}=$ absolute permeability

$p \quad=$ pressure

$q \quad=$ flux

$\vec{u} \quad=$ velocity

$\vec{x} \quad=$ spatial coordinate

$f \quad=$ volumetric rate

$\mu \quad=$ viscosity

$\tilde{\mu} \quad=$ effective viscosity

Domain and grid:

$\Omega \quad=$ entire physical domain

$\partial D \quad=$ boundary of domain $D$

$E \quad=$ cell in the fine grid

$\Omega_{i}=$ coarse block number $i$

$\Omega_{i j} \quad=$ support for basis function $\vec{\psi}_{i j}$

Basis functions, etc:

$\vec{v} \quad=\quad$ test function for velocity on fine scale

$\pi \quad=$ test function for pressure on fine scale

$\vec{\psi}_{i j} \quad=$ basis function on interface of block $i$ and $j$

$\vec{\psi}_{i j}^{H} \quad=$ hybrid split of basis function

$\phi_{i j}=$ pressure associated with $\vec{\psi}_{i j}$

$w_{i} \quad=$ weight function associated with coarse block $\Omega_{i}$
Vectors and matrices:

$\boldsymbol{p} \quad=\quad$ vector of cell/block pressures

$\boldsymbol{\lambda}=$ vector of face pressures

$\boldsymbol{u}=$ vector of outward velocities on cell/block faces

$\boldsymbol{q}=$ vector of outward fluxes on cell/block faces

$\boldsymbol{B}=$ inner product of velocity basis functions

$C \quad=$ integral of divergence of velocity b.f.

$\boldsymbol{D} \quad=$ mapping from local to global faces numbering

$\boldsymbol{T}_{E}=$ transmissibility matrix for cell $E$

$\boldsymbol{\Psi}=$ matrix of all basis functions

$\boldsymbol{A}=$ matrix of all face areas

Numbers:

$N \quad=$ number of cells in fine grid

$N_{i} \quad=$ number of cells in coarse block $\Omega_{i}$

Subscripts:

$i, j, k=$ block/cell numbers

$D, S=$ Darcy and Stokes

$S B=$ Stokes-Brinkman

Superscripts:

$c=$ coarse/fine grid

$R T=$ Raviart-Thomas

$T H=$ Taylor-Hood

\section{References}

Aarnes, J. E. 2004. On the use of a mixed multiscale finite element method for greater flexibility and increased speed or improved accuracy in reservoir simulation. Multiscale Model. Simul., 2(3):421-439 (electronic).

Aarnes, J. E., Krogstad, S., and Lie, K.-A. 2006. A hierarchical multiscale method for two-phase flow based upon mixed finite elements and nonuniform coarse grids. Multiscale Model. Simul., 5(2):337-363 (electronic).

Aarnes, J. E., Krogstad, S., and Lie, K.-A. 2008. Multiscale mixed/mimetic methods on corner-point grids. Comput. Geosci., 12(3):297-315. Doi:10.1007/s10596-007-9072-8.

Arbogast, T. and Brunson, D. S. 2007. A computational method for approximating a Darcy-Stokes system governing a vuggy porous medium. Comput. Geosci., 11(3):207-218.

Arbogast, T. and Bryant, S. L. 2002. A two-scale numerical subgrid technique for waterflood simulations. SPE J., 7(4):446-457.

Arbogast, T. and Gomez, M. S. M. 2009. A discretization and multigrid solver for a Darcy-Stokes system of three dimensional vuggy porous media. Comput. Geosci. Doi: 10.1007/s10596-008-9121-y.

Arbogast, T. and Lehr, H. L. 2006. Homogenization of a Darcy-Stokes system modeling vuggy porous media. Comput. Geosci., 10(3):291-302.

Brezzi, F. and Fortin, M. 1991. Mixed and hybrid finite element methods, volume 15 of Springer Series in Computational Mathematics. Springer-Verlag, New York.

Chen, Z. and Hou, T. 2003. A mixed multiscale finite element method for elliptic problems with oscillating coefficients. Math. Comp., 72:541-576.

Christie, M. A. and Blunt, M. J. 2001. Tenth SPE comparative solution project: A comparison of upscaling techniques. SPE Reservoir Eval. Eng., 4:308-317. url: http: //www.spe.org/csp/.

Hajibeygi, H., Bonfigli, G., Hesse, M. A., and Jenny, P. 2008. Iterative multiscale finite-volume method. J. Comput. Phys, 227(19):8604-8621.

Hou, T. and Wu, X.-H. 1997. A multiscale finite element method for elliptic problems in composite materials and porous media. J. Comput. Phys., 134:169-189.

Jenny, P., Lee, S. H., and Tchelepi, H. A. 2003. Multi-scale finite-volume method for elliptic problems in subsurface flow simulation. J. Comput. Phys., 187:47-67. 
Karper, T., Mardal, K.-A., and Winther, R. 2009. Unified finite element discretizations of coupled Darcy-Stokes flow. Numer. Meth. PDEs, 25(2):311-326. Doi: 10.1002/num.20349.

Kippe, V., Aarnes, J., and Lie, K.-A. 2008. A comparison of multiscale methods for elliptic problems in porous media flow. Comput. Geosci., 12(3):377-398. Doi: 10.1007/s10596-007-9074-6.

Krogstad, S., Lie, K.-A., Nilsen, H. M., Natvig, J. R., Skaflestad, B., and Aarnes, J. E. 2009. A multiscale mixed finite-element solver for threephase black-oil flow. Paper SPE 118993, presented at SPE Reservoir Simulation Symposium, The Woodlands, TX, USA, 2-4 February.

Lee, S. H., Wolfsteiner, C., and Tchelepi, H. 2008. Multiscale finite-volume formulation for multiphase flow in porous media: black oil formulation of compressible, three phase flow with gravity. Comput. Geosci., 12(3):351-366. Doi:10.1007/s10596-007-9069-3.

Natvig, J. R., Skaflestad, B., Bratvedt, F., Bratvedt, K., Lie, K.-A., Laptev, V., and Khataniar, S. K. 2009. Multiscale mimetic solvers for efficient streamline simulation of fractured reservoirs. Paper SPE 119132, presented at 2009 SPE Reservoir Simulation Symposium, The Woodlands, Texas, USA, 2-4 February.

Nordbotten, J. 2009. Adaptive variational multiscale methods for multiphase flow in porous media. Multiscale Model. Simul., 7(3):1455-1473.

Popov, P., Bi, L., Efendiev, Y., Ewing, R. E., Qin, G., Li, J., and Ren, Y. 2007. Multi-physics and multi-scale methods for modeling fluid flow through naturally-fractured vuggy carbonate reservoirs. Paper SPE 105378, presented at $15^{\text {th }}$ SPE Middle East Oil \& Gas Show and Conference, Bahrain, 11-14 March.

Popov, P., Efendiev, Y., and Qin, G. 2009. Multiscale modeling and simulations of flows in naturally fractured karst reservoirs. Commun. Comput. Phys., 6:162-184.

Skaflestad, B. and Krogstad, S. 2008. Multiscale/mimetic pressure solvers with near-well grid adaption. In Proceedings of ECMOR XI - 11th European Conference on the Mathematics of Oil Recovery, number A36, Bergen, Norway. EAGE.

Wu, Y.-S., Qin, G., Ewing, R. E., Efendiev, Y., Kang, Z., and Ren, Y. 2006. A multiple-continuum approach for modeling multiphase flow in naturally fractured vuggy petroleum reservoirs. Paper SPE 104173, presented at SPE International Oil \& Gas Conference and Exhibition, Beijing, China, 5-7 December.

Zhou, H. and Tchelepi, H. 2008. Operator-based multiscale method for compressible flow. SPE J., 13(2):267-273. 